\title{
Dictynna
}

Dictynna

Revue de poétique latine

13 | 2016

Varia

\section{Il lamento di Neobule (carm. 3.12): un'eroide oraziana?}

\section{Gianpiero Rosati}

\section{Q OpenEdition}

\author{
Journals
}

Edizione digitale

URL: http://journals.openedition.org/dictynna/1300

DOI: 10.4000/dictynna.1300

ISSN: 1765-3142

Notizia bibliografica digitale

Gianpiero Rosati, « II lamento di Neobule (carm. 3.12): un'eroide oraziana? », Dictynna [En ligne], 13।

2016, mis en ligne le 20 décembre 2016, consulté le 11 septembre 2020. URL : http://

journals.openedition.org/dictynna/1300; DOI : https://doi.org/10.4000/dictynna.1300

Questo documento è stato generato automaticamente il 11 settembre 2020.

\section{(c) (i) (9)}

Les contenus des la revue Dictynna sont mis à disposition selon les termes de la Licence Creative Commons Attribution - Pas d'Utilisation Commerciale - Pas de Modification 4.0 International. 


\title{
Il lamento di Neobule (carm. 3.12): un'eroide oraziana?
}

\author{
Gianpiero Rosati
}

\section{Modello alcaico e problema della voce}

\begin{abstract}
Miserarum est neque amori dare ludum neque dulci
mala vino lavere, aut exanimari

metuentis patruae verbera linguae.

Tibi qualum Cythereae puer ales, tibi telas operosaeque Minervae studium aufert,

Neobule, Liparaei nitor Hebri,

simul unctos Tiberinis umeros lavit in undis,

eques ipso melior Bellerophonte,

neque pugno neque segni pede victus,

catus idem per apertum fugientis agitato

grege cervos iaculari et celer arto

latitantem fruticeto excipere aprum.

[È triste non giocare all'amore né sciogliere nel dolce vino gli affanni, o svenire atterrite ai colpi vibrati dalla lingua d'uno zio paterno. Dal cesto delle lane il figlio alato di Citerea ti distoglie, o Neobule, dalle tele e dall'opera attenta di Minerva lo splendore di Ebro lipareo, quando bagna le lucenti spalle nelle onde tiberine, cavaliere più abile dello stesso Bellerofonte, e invincibile nel pugilato o nella corsa, esperto nel trafiggere i cervi in fuga sulla pianura in trepido branco, e rapido nel cogliere il cinghiale che si cela nell'intricata boscaglia (trad. L. Canali)]
\end{abstract}

1 Sono molte le ragioni che spiegano la particolarità, all'interno del corpus lirico oraziano, dell'ode 3.12, direi anzi la sua unicità, e che giustificano quindi la sua assoluta marginalità negli studi oraziani. ${ }^{1}$ L'attenzione che gli interpreti di Orazio lirico, e di Orazio tout court, le hanno dedicato è davvero scarsa, e ad essa corrisponde una valutazione quasi sempre riduttiva, come di un freddo esercizio letterario, uno 'studio' alla maniera di Alceo. 
2 La prima di queste ragioni è nella eccezionalità del metro: lo ionico a minore, una sequenza di due brevi più due lunghe (che corrisponde alla prosodia del nome stesso di Neobule, la protagonista), in una successione di quaranta metri, organizzati per gruppi di dieci ionici in sequenza (cioè quattro decametri). Nonostante la poesia latina conosca la presenza di metri ionici in varie combinazioni, un sistema di ionici puri non è mai attestato altrove; e la scelta di un metro così eccezionale non è ovviamente priva di significato. Come gli interpreti hanno più volte rilevato, l'ethos che questo metro esprime è il più adatto a dare voce al lamento di una donna sulla propria condizione di vita. "Contenuto e metro concordano meravigliosamente: nessun'altra di tutte le altre forme metriche oraziane corrisponderebbe al corso monotono e uniforme dei pensieri di una fanciulla solitaria come questa sequenza ininterrotta e indifferenziata di ionici puri":" una sorta di chanson de toile, quindi, che nella monotonia del ritmo (che dagli antichi viene percepito come "molle e rallentato": un ritmo insomma 'femminile') ${ }^{3}$ riflette la monotonia della vita della sua protagonista.

3 Sull'interpretazione dell'ode ha certamente gravato anche il problematico rapporto con il suo modello greco, il quale, già fin da Bentley, è stato individuato in Alceo (frg. 10 Voigt): data l'estrema frammentarietà in cui questo testo ci è noto (un verso di incipit trasmesso da Efestione cui sono stati aggiunti altri due frammenti di tradizione indiretta), il suo rapporto con l'ode oraziana è quanto mai difficile da definire. Anche se oggi si registra una giusta reazione contro le reciproche interferenze nell'interpretazione dei due testi - troppo spesso in passato si è interpretata l'ode di Orazio alla luce del frammento di Alceo (Wilamowitz arrivava addirittura a parlare di “Übersetzung"), ${ }^{4}$ e quest'ultimo alla luce dell'ode oraziana -, è indubbio che di modello, in qualche modo, si deve trattare (almeno nel senso di modello-codice, se non proprio modello-esemplare). Il frammento, anch'esso in decametri ionici, inizia con una voce di donna (in questo caso è chiaro da subito che si tratta di una voce femminile) che lamenta la propria infelicità:

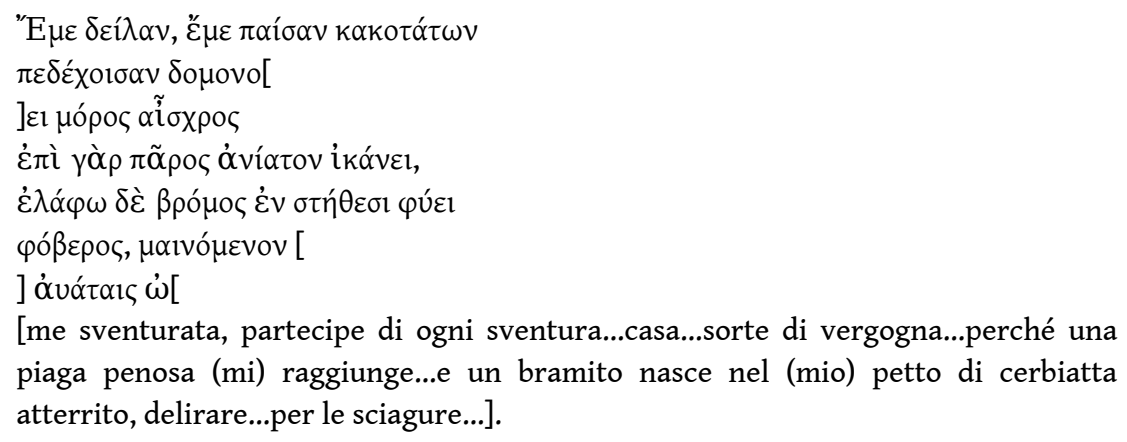

4 La causa di questo dolore non è esplicita: può essere un lamento generico per la condizione femminile in sé, o motivato da ragioni specifiche ma per noi oscure. In ogni caso è opportuno non costruire l'interpretazione dell'ode oraziana sulla base del frammento alcaico; quand'anche riuscissimo a definirne meglio i contenuti e il tono, nulla autorizzerebbe ad appiattire l'ode oraziana sul testo-modello, che può essere stato un puro 'pre-testo'. Sarà più giustificato, come faceva Pasquali, ${ }^{5}$ riconoscervi una gnome iniziale che, come tante altre volte in Orazio, non condiziona poi in maniera vincolante il resto del componimento.

5 Il problema del rapporto con Alceo porta con sé anche quello della voce, di chi nell'ode parla. Diversamente che in Alceo l'ode inizia infatti senza che questo sia chiaro: solo al centro del componimento, al v. 6 (nella colometria qui adottata), ${ }^{6}$ l'apostrofe a Neobule, 
preparata dall'anafora del tibi, introduce (insieme al nome di Ebro, l'oggetto della passione) il nome dell'interlocutore di chi parla. Che a parlare sia il poeta pensava ad es. lo Pseudo-Acrone (Ad Neobulem amicam scribit amantem Hebrum adulescentem), e più di recente, tra vari altri, Viktor Pöschl, ${ }^{7}$ Francis Cairns $^{8}$ e Mario Citroni ("è quasi certamente da intendere come pronunciata dal poeta e rivolta alla donna"), il quale osserva che il lettore sa che quando nelle odi vi è un io parlante è di norma quello del poeta, a meno che - aggiunge - sovrapponendo alla lettura di quest'ode il ricordo di quella di Alceo il lettore potesse capire che qui si trattava di un monologo del personaggio. ${ }^{9}$ Che a parlare sia la stessa Neobule non si può infatti escludere, ${ }^{10} \mathrm{e}$ potremmo quindi trovarci di fronte a un suo monologo, appunto come in Alceo. D'altra parte anche quello di Alceo non può essere stato un monologo in senso proprio (cantato cioè nel simposio da una donna, che non vi era ammessa), ma dev'essere un procedimento di persona loquens, un quadro cioè di genere, una canzone a soggetto, che faceva parte del repertorio ed ebbe larga fortuna nella letteratura ellenistica. ${ }^{11}$

Gli studiosi di Orazio tendono comunque a non fare della esatta identificazione della voce parlante un problema tale da inibire il senso del componimento: anche se a parlare è Orazio, di fatto "l'ode gravita tutto intorno all'interlocutrice e non differisce in nulla da un monologo dell'interlocutrice di cui il poeta si faccia interprete". ${ }^{12}$ Anche se insomma non sono gli occhi stessi di Neobule a darci la sua visione del mondo, sarebbe Orazio ad assumere i suoi occhi di donna e a dar voce ai sentimenti di lei. Altri preferiscono vedere in questa scelta una buona dose di consapevolezza ironica e di scetticismo, ${ }^{13}$ ma anziché interrogarsi sulla autenticità di questa voce conviene forse chiedersi perché Orazio adotta questa strategia, perché vuole lasciare indistinta la voce parlante (un aspetto su cui torneremo più avanti).

7 Si può insomma capire, per questa difficoltà di fissare punti fermi, su cui fondare l'interpretazione, il disagio degli interpreti, e la riluttanza ad occuparsi di quest'ode sfuggente, strana anche nella struttura e che lascia come un'impressione generale di incompiutezza. ${ }^{14}$

\section{Mondo greco e mondo romano}

Un aspetto rilevante dell'ode è il suo carattere culturalmente ibrido, che unisce tratti del mondo greco ad altri che sono evidentemente di quello romano. Sui tratti romani insiste ad es. già Pasquali,${ }^{15}$ che ha interesse ad accentuare il carattere romano dell'ode per mostrare come Orazio, preso lo spunto da Alceo, faccia poi poesia propria, autonoma, moderna e romana. Sui tratti greci - già vistosi nella ripresa di un'ode di Alceo, e di un nome, Neobule, che è quasi un marchio di Archiloco - invece insiste molto Francis Cairns ${ }^{16}$ il quale li vede specialmente nella caratterizzazione di Ebro, una figura di efebo che deve molto alla lirica greca arcaica: la rappresentazione della bellezza fisica di Ebro, del suo corpo lucente dopo gli esercizi atletici, ha corrispondenze significative con passi di Pindaro (spec. Pyth. 9, 97-103), ma Cairns cerca di andare anche oltre Pindaro, per ritrovare nella lirica arcaica lo schema stesso della situazione oraziana (di cui segnala una traccia, per la verità piuttosto labile, in Alcmane). A me pare invece che la scena-modello più pertinente per l'immagine del corpo di un atleta che seduce chi lo guarda sia da cercare appunto in poesia ellenistica: penso ad es., in Callimaco, al fascino esercitato da Aconzio dopo il bagno (frg. 68 Pf.) e soprattutto, come nota già Cairns, al secondo idillio di Teocrito (Le incantatrici, vv. 77-82, dove 
Simeta racconta di aver visto Delfi uscire dalla palestra e di essersene innamorata di

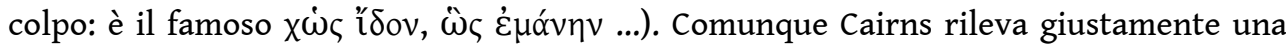
forte impronta greca nell'ambientazione e nella caratterizzazione dei personaggi, nell'ethos complessivo, nei loro stessi nomi: Neobule, Lipareus, Hebrus, Cytherea, Bellerophontes. ${ }^{17}$

Ciò che invece a me pare assai meno convincente è la sua interpretazione dell'ode: Cairns ha ragione a cogliere in Orazio un atteggiamento 'simpatetico' verso Neobule, ma già più forzato mi pare vedere questo atteggiamento verso lo stesso Ebro, del quale sarebbe apprezzata sia la bellezza che il pudore: Orazio darebbe così la sua approvazione alla "scelta matrimoniale" di Neobule: lei, una casta vergine romana in età da matrimonio innamorata di un virtuoso giovane romano. Secondo Cairns, l'ode avrebbe quindi una funzione morale e sociale, addirittura di sostegno al programma morale del regime augusteo, incoraggiando il matrimonio fra due giovani romani. ${ }^{18}$

Per quanto riguarda invece i tratti marcatamente romani, va anzitutto rilevato il riferimento a una famosa proibizione della cultura romana, il divieto alle donne di bere vino (divieto fatto risalire fin alle origini di Roma, e che dovette dissolversi fra tarda repubblica e impero; non sappiamo di un analogo divieto per le donne greche) e meno probabilmente - almeno secondo un'interpretazione controversa - all'altrettanto famoso, o piuttosto famigerato, istituto della cultura romana arcaica connesso (anche se non originariamente) a quella proibizione, cioè il cosiddetto ius osculi (un diritto che qui eserciterebbe il patruus). ${ }^{19}$

11 È evidente insomma il carattere romano delle costrizioni sociali di cui Neobule si sente vittima: alla cultura romana ci richiama soprattutto l'arcigna figura del patruus, lo zio paterno (di cui è nota l'importanza nel sistema romano delle relazioni di parentela), ${ }^{20}$ un'antonomasia di severità e durezza, specialmente nella repressione del lusso e del piacere amoroso. Il patruus suggerisce non solo l'autorità, ma l'autorità specificamente maschile, e dunque il senso del genus patrilineare, con la subordinazione della donna al suo controllo spietato, tale da suscitare quasi terrore (exanimari metuentis). A questa presenza maschile opprimente e minacciosa si oppone un'altra figura maschile ammirata e desiderata ma assente, estranea all'orizzonte di vita della donna, che la può solo guardare e sognare da lontano. L'esistenza femminile di Neobule si consuma nella tensione fra queste due uniche figure maschili reciprocamente incompatibili, emblemi rispettivamente della proibizione e del desiderio: nel suo mondo di donna vittima di un ferreo controllo sociale non ci sono altre presenze o relazioni.

L'ambientazione dell'ode è dunque romana, ma questa totale assenza di socialità riconduce soprattutto al mondo greco (è noto che la condizione della donna romana da questo punto di vista è meno rigida), così come greca è la situazione di carattere simposiale invidiata agli uomini, che evoca un ambiente e un'atmosfera 'alcaica' (l'idea del vino come sollievo degli affanni implica un'idea di socialità maschile). Sembra insomma che Orazio abbia voluto riscrivere in versione romana il lamento di una fanciulla greca innamorata, innestando sul confronto fra mondo greco e mondo romano anche un confronto di genere sessuale, fra mondo femminile e mondo maschile, rappresentato come vedremo anche attraverso una concettualizzazione spaziale dei ruoli, cioè attraverso un'opposizione fra immobilità e chiusura del mondo interno (femminile) da un lato, e agilità e apertura del mondo esterno (maschile) dall'altro. 


\section{Un confronto di gender}

13 Il problema del genere sessuale è già posto nella prima parola, miserarum. Miser è davvero parola-chiave: in primo luogo definisce la tonalità dominante del componimento, la sofferenza (e insieme orienta anche sulle cause di quella sofferenza: da Catullo ${ }^{21}$ in poi miser è l'aggettivo degli amori infelici, non corrisposti), mentre il femminile designa da subito il genere che il discorso coinvolge. Il plurale poi (che contrasta con l'iniziale ع̌ $\mu \varepsilon$ del modello alcaico) a sua volta generalizza il discorso alla condizione di tutte le donne (che non possono amare liberamente, e nemmeno ricorrere al vino come conforto alle pene d'amore), prima che la seconda strofa con tibi restringa il campo e individualizzi il discorso in rapporto a Neobule.

La prima parola ci dà quindi una serie di indicazioni essenziali, mentre la prima struttura sintattica, miserarum est, il genitivo 'di convenienza' (come lo chiamano le grammatiche), ci dice un destino, una condanna ai vincoli appunto della convenienza e dei doveri sociali, che viene successivamente definita in negativo dall'anafora di neque, la quale insiste sulle privazioni, sui divieti che mortificano l'esistenza femminile.

Se il primo divieto riguarda espressamente l'amore, la facoltà di assecondare liberamente la passione come possono fare i maschi (dare ludum è locuzione della lingua parlata ${ }^{22}$ il registro espressivo adeguato alla naturale spontaneità del comportamento maschile), anche il secondo, il conforto del vino per 'lavar via' i mali, gli affanni, sembra rinviare alla sfera delle delusioni d'amore, ${ }^{23}$ di cui Bacco è un remedium tradizionale, specie nell'elegia (e qui infatti il registro espressivo si innalza con la metafora dulci mala vino lavere, che è ricercata e senza paralleli). ${ }^{24}$ Ciò che Neobule invidia agli uomini è quindi la piena libertà di comportamento nella sfera amorosa, la possibilità di vivere le proprie scelte senza inibizioni di sorta.

16 Ma chi è Neobule? qual è la sua età? e in che tipo di società vive? Pasquali la dice una "fanciulla romana di buona famiglia", ma rileva come i divieti che la amareggiano sembrino orientare verso uno stile di vita arcaico, fin troppo severo rispetto all'età di Orazio; uno zio quindi anche 'passatista', più arcaico dell'età in cui vive. In realtà è difficile identificare un contesto socio-culturale in cui collocare questa fanciulla; perché accanto agli elementi di cultura romana (il divieto del vino, la severità dello zio, gli esercizi ginnici e la caccia, attività in voga fra i giovani romani del tempo) molti altri rinviano piuttosto alla cultura greca, a cominciare ovviamente dai nomi, Neobule e Ebro. È alla cultura letteraria greca, ad es., che rinvia il motivo della fanciulla innamorata la quale non riesce più a dedicarsi ai suoi lavori di donna, motivo che, già

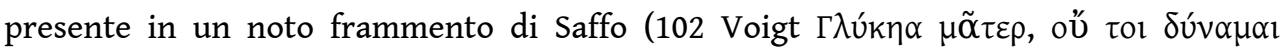

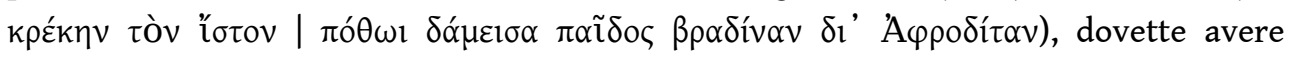
fortuna specie nella letteratura d'età ellenistica (come provano anche le molte testimonianze latine $)^{25}$ così come nelle arti figurative. ${ }^{26} \mathrm{E}$ alla cultura letteraria ellenistica riconduce lo stesso lamento di una donna innamorata, che richiama ovviamente il secondo idillio di Teocrito nonché il cosiddetto Fragmentum Grenfellianum, ${ }^{27} \mathrm{ma}$ anche (come aveva ben visto Heinze) il tema del confronto fra la condizione di vita femminile e quella maschile, che ha sì lontane radici euripidee ma si sviluppa soprattutto in età ellenistica, e su cui torneremo più sotto.

Vediamo più in dettaglio come si articola nell'ode questo confronto. Dopo le premesse generali sulla condizione femminile nella prima strofa, la seconda strofa definisce l'orizzonte di vita individuale di Neobule: lo caratterizzano il lavoro come dovere 
(qualum... telas operosaeque / Minervae studium), l'assenza di socialità, l'idea di un mondo chiuso. Di lei sappiamo ciò che non è e non può essere, i suoi desideri frustrati: l'unica notizia in positivo che di lei abbiamo è che passa il tempo al telaio.

Dopo le prime due strofe sull'esistenza chiusa e oscura di Neobule irrompe nelle due strofe finali lo 'splendore' di Ebro: Liparaei, che designa la provenienza dalle isole Lipari,

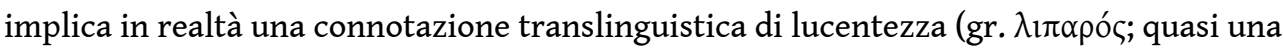
conferma sul piano linguistico di quel 'confronto' culturale greco/romano che percorre tutta l'ode; così come operosa, detto di Minerva, e mai altrove riferito a un dio, è il calco latino del greco غ́pүóv $\eta$, l'epiteto di Atena). Una lucentezza (ricordo fra l'altro che Lipari è la sede della fucina di Efesto-Vulcano; e quindi facilmente associata all'idea del fuoco) che è accentuata da nitor ma anche da unctos, creando come un'aureola che avvolge questo giovane eroe. ${ }^{28}$ La comparsa di Ebro è come un'epifania divina, un'apparizione improvvisa (simul insiste appunto sull'immediatezza dell'effetto), e questo alone luminoso accompagna la serie di aristie sportive in cui egli manifesta la sua eccellenza.

19 A questa caratterizzazione contribuisce naturalmente anche la dichiarazione di superiorità su Bellerofonte, l'eroe che cavalca Pegaso e che uccide la Chimera, e noto per tante altre imprese belliche. Io non credo che il paragone intenda suggerire la castità di Ebro, ${ }^{29}$ ma che sia motivato piuttosto dal fatto che Bellerofonte è un paradigma di seduzione esercitata su una donna, Stenebea (cfr. nello stesso Orazio, carm. 3.7.13-16, dove ha una funzione simile). Credo cioè che Orazio si serva del paragone come per far affiorare (agli occhi del lettore) quello che è un pensiero rimosso, censurato, di Neobule: il richiamo a Bellerofonte assimila in qualche modo Neobule a Stenebea, la regina sedotta dalla sua bellezza e vanamente innamoratasi di lui, ed evoca quindi un'idea di amore deluso, frustrato, e anche dalle conseguenze tragiche per effetto di calunnie (Neobule potrebbe sentirsi cioè un po' nella condizione di Stenebea). Al tempo stesso l'evocazione di Bellerofonte conferisce a Ebro, il cui fascino viene detto ancora maggiore, l'aura del mito, e la leggerezza trionfale della sua comparsa sulla scena lo assimila al cythereae puer ales, a Eros, facendo della loro quasi un'azione congiunta: ${ }^{30}$ l'anafora del tibi insiste sulla simmetria delle loro due azioni, che sono poi la stessa azione nei confronti di Neobule, quella di sconvolgerne la quieta esistenza operosa togliendole l'uno materialmente il qualum (un'immagine che richiama interventi scherzosi di Eros come nel terzo libro di Apollonio), l'altro psicologicamente lo studium, distogliendone cioè l'interesse dai lavori femminili.

Per indicare l'eccellenza di Ebro in tutte le prove atletiche il poeta ricorre all'anafora neque... neque (v. 9 neque pugno neque segni pede victus) già impiegata al v. 1, marcando quindi l'opposizione fra la condizione maschile e quella femminile: mentre lì insisteva sulla totalità delle privazioni della donna ("non questo... non quello..."), qui esprime il senso della pienezza di vita maschile, che non conosce invece limitazioni ("non è secondo né in questo né in quello...").

21 Nuoto, equitazione, pugilato, corsa, caccia (una sorta di pentathlon alla romana, che aveva luogo sia lungo le rive del Tevere sia nel Campo Marzio sia nelle campagne attorno alla città) sono pratiche che implicano socialità e competizione (victus), e si svolgono comunque in un universo unicamente maschile, che esclude la presenza femminile - direi programmaticamente, dato il proverbiale antagonismo fra attività ginnico-sportive e eros (non parlo qui di convenzioni letterarie ${ }^{31}$ ma di valori eticosociali). ${ }^{32}$ 


\section{Voce femminile e poesia ellenistico-augustea} movenza di una fantasticheria femminile: ${ }^{33}$ un sogno ad occhi aperti che si protrae in dissolvenza e su cui l'ode si chiude (in una sorta di 'feminine ending' come quelli analizzati da Ellen Oliensis). ${ }^{34}$ Dopo la scena 'chiusa' iniziale (che rappresenta la mortificazione di un'esistenza inibita e in preda alla paura) della prima strofa, e l'effetto di 'fermo', di inazione della seconda strofa, le ultime due producono invece un effetto di 'aperto' (10 per apertum) e di 'movimento' (vv. 8-9 eques... neque segni pede; 10 fugientis agitato), di dispiegamento di un'esistenza ricca e articolata, in un orizzonte libero e naturale (lo stesso nome di Ebro, il principale fiume della Tracia, oggetto di un'altra ode di Alceo che lo celebra come il più bello dei fiumi [frg. 45 Voigt], suggerisce l'idea di una forza naturale incontenibile). ${ }^{35}$

L'aristia venatoria di Ebro consiste nel cacciare cervi e cinghiali, stanando le sue vittime e costringendole a uscire allo scoperto (il cinghiale 'si nasconde' [12] nello spazio chiuso e stretto [arto] della boscaglia, un po' come Neobule vive nel chiuso dello spazio domestico): Ebro si afferma insomma come un signore della forza dispiegata nello spazio aperto, la sua irruzione sulla scena sconvolge la tranquilla vita del mondo animale così come quella operosa e solitaria della povera Neobule, anch'essa vittima del potere di lui.

L'ode finisce senza una conclusione: che l'amore per Ebro trovi soddisfazione, come pensa Cairns, è una petizione di principio: nulla dice che il desiderio di Neobule sia appagato. L'impressione che resta al lettore è quella di una mente che continua a fantasticare come all'inizio del componimento, suggerendo l'idea di una tensione che permane irrisolta, e di un'esistenza senza sviluppi o vie di uscita. ${ }^{36}$

Il nome stesso di Neobule sembrerebbe escludere un esito positivo (in effetti non è stato chiarito finora il nesso fra il nome e l'interpretazione generale dell'ode). ${ }^{37} \mathrm{~A}$ me pare indubitabile l'associazione con Archiloco, e con i suoi giambi (i suoi verbera) ${ }^{38}$ scagliati contro Licambe e la figlia; ${ }^{39} \mathrm{e}$ in questo senso anche la cattiva reputazione di cui la donna attaccata dal poeta ebbe notoriamente a soffrire potrebbe avere un collegamento con i timori di Neobule per i verbera linguae dei moralisti. Il suo si configura quindi come un futuro senza molte speranze, soprattutto se si pensa che, secondo la tradizione, Neobule si sarebbe suicidata, vittima dell'azione congiunta di un padre autoritario e di un pretendente deluso che si vendica diffamandola ${ }^{40} \mathrm{e}$ condannandola a morte: il nome stesso di Neobule evoca quindi un'esistenza femminile schiacciata dal potere complice di una società di maschi.

Che l'ode sia un'ode d'amore, il lamento di un'innamorata per l'impossibilità di 'dare corso' alla sua passione, a me pare indubbio; e tuttavia non è solo questo. Come dicevo, è anche una riflessione dolente, condotta dal punto di vista di una donna, sulla differenza sessuale, sulla diversa condizione delle donne rispetto agli uomini: il miserarum iniziale riguarda le donne in quanto tali, tutte le donne - quelle ovviamente a cui Neobule si assimila, le donne libere, della buona società, destinate a diventare matrone, e quindi tenute a una condotta irreprensibile. Alla libertà e alla pienezza della vita di Ebro (sport e caccia, cioè socialità e competizione) si oppone la chiusura e la limitazione di quella di Neobule, costretta a condurre un'esistenza soggetta al dovere ( $\mathrm{i}$ lavori domestici, la reputazione da tutelare, la rinuncia ai conforti del vino) e a sognare 
ad occhi aperti 'guardando vivere' gli uomini (cioè Ebro, un'antonomasia di uomo felice e realizzato).

Se la si considera una riflessione sulla condizione delle donne e sulla loro psicologia, l'ode 3.12 sembra un unicum nella poesia oraziana, sul piano tematico oltre che formale. Un esperimento quindi, che risulta però meno eccezionale se visto nel quadro della poesia augustea, quella elegiaca in particolare, e sullo sfondo della poesia ellenistica. La mimesi della voce femminile (o comunque la lettura da parte maschile della psiche della donna) istituisce infatti un rapporto con l'elegia, il genere letterario che più di ogni altro a Roma presta attenzione alla figura e alla psiche della donna: che la pone al centro dell'universo poetico esplorando o piuttosto costruendo l'identità femminile. L'adozione di un'ottica femminile fa pensare al quarto libro di Properzio (Aretusa, Cornelia, Tarpea, la lena Acantide, e ovviamente la stessa Cinzia), alle Heroides ovidiane (l'elegia messa in bocca alle donne, vista con gli occhi delle donne) e soprattutto a Sulpicia. ${ }^{41}$

28 L'elegia latina è infatti un genere che, come ha scritto Maria Wyke, "interrogates Roman culture's construction of masculinity and femininity", ${ }^{42} \mathrm{e}$ richiama l'attenzione alle tensioni e fluidità della differenziazione di genere. Tutto ciò, oltre ad essere la spia di un interesse a 'simulare l'altro' (quello che William Fitzgerald chiama 'drama of position'), ${ }^{43}$ rivela comunque un interesse nuovo per la categoria del gender in letteratura, un'attenzione alla personalità e alla psicologia della donna, di cui la cultura letteraria romana (maschile) tenta un approfondimento con i suoi stessi occhi, fino a simulare la sua stessa voce. Questo può naturalmente esser visto come un processo di 'colonizzazione' da parte della cultura maschile dominante di un territorio appunto nuovo e inesplorato, di cui si definiscono i confini e si assume il controllo (come Pigmalione che 'si costruisce' la sua donna); ${ }^{44}$ e tuttavia, come ha osservato Froma Zeitlin a proposito dell'assunzione della voce femminile da parte del poeta (maschile) nel teatro attico, anche se in quella voce il poeta, come un ventriloquo, finisce per riaffermare le strutture dell'autorità maschile, il primo effetto di questo fenomeno è comunque di allargare l'idea maschile dell'universo, aprirlo a dimensioni ed emozioni finora sconosciute o rimosse. ${ }^{45}$ Costruire un soggetto femminile capace di interrogarsi, di riflettere su di sé e sulla propria differenza, significa anche sviluppare la capacità di interrogarsi e riflettere sulla propria natura da parte della cultura maschile. Rappresentare 'il femminile' è insomma anche una forma di introspezione, di introspezione del mondo maschile. Ma non ci interessa qui tanto un giudizio di merito su questa operazione e sulle sue implicazioni di potere culturale e sociale; ci basta rilevare un fenomeno importante nella storia della cultura letteraria antica come cornice entro la quale anche quest'ode oraziana può trovare un suo spazio.

Quello della sperimentazione della voce femminile è un capitolo della poesia ellenistica su cui la nostra documentazione è molto lacunosa, ma che abbiamo motivo di credere fosse assai più sviluppato e articolato. Vi rientrano due testi già richiamati, l'idillio $2 \mathrm{di}$ Teocrito (il monologo di una donna, Simeta, che racconta all'ancella la storia del suo amore sfortunato) e il cd. Fragmentum Grenfellianum, noto come 'Lamento della donna abbandonata' di fronte alla porta chiusa dell'amato (una sorta di paraklausithyron alla rovescia, come appare a noi che siamo abituati alla prospettiva maschile dell'elegia latina) ${ }_{,}^{46}$ ma vi si possono ricondurre anche alcuni componimenti dell' Anthologia Palatina e altri testi ancora. 
30 Ma c'è infine un ulteriore aspetto che in qualche modo collega l'ode oraziana all'elegia latina, in particolare a Ovidio, e attraverso di essa a un tema che deve aver avuto un certo rilievo nella cultura letteraria ellenistica, del quale troviamo le tracce in un passo appunto di Ovidio (non a caso nelle Heroides, in una delle doppie, in cui il confronto femminile vs maschile è in qualche modo iscritto nel testo stesso), nel poemetto, dedicato allo stesso mito, del tardo poeta greco Museo (fine V sec.), e in un epigramma del bizantino Agazia (VI sec.). Così l'ovidiana Ero, scrivendo all'amato Leandro, argomenta le ragioni della sua impazienza con l'inazione cui è condannata l'esistenza femminile (her. 19.7-14):

Ut corpus, teneris ita mens infirma puellis:

deficiam, parvi temporis adde moram!

Vos modo venando, modo rus geniale colendo

ponitis in varia tempora longa mora.

Aut fora vos retinent aut unctae dona palestrae,

flectitis aut freno colla sequacis equi;

nunc volucrem laqueo, nunc piscem ducitis hamo;

diluitur posito serior hora mero.

[Come il corpo, anche l'anima è fragile nelle tenere fanciulle; aggiungi solo un breve ritardo, ed io morirò. Voi uomini, ora nella caccia, ora coltivando i campi fecondi, consumate lungo tempo in varie attività. Vi trattiene il foro o gli esercizi della lucente palestra o piegate col morso il collo del cavallo rendendolo docile; ora prendete ol laccio gli uccelli, ora i pesci con l'amo; e col vino davanti fate passare le ore tarde].

31 Il tema sarà brevemente ripreso dalla voce della protagonista nel poemetto Ero $e$ Leandro del greco Museo (191 s.):

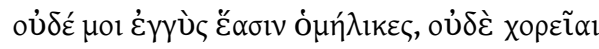

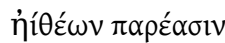

[non ho accanto a me coetanee né danze di giovani]

e tornerà in maniera più diffusa in un componimento del bizantino Agazia (AP 5.297):

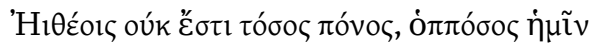

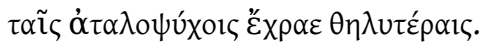

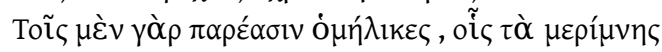

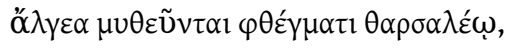

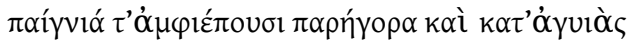

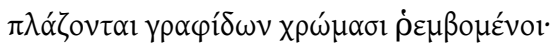

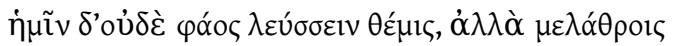

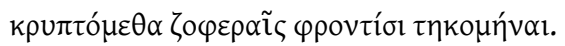

[Ai ragazzi non toccano tutte quante le pene

che sono toccate in sorte a noi povere donne.

Hanno amici della stessa età, ai quali confidano

i loro dolori ed affanni con voce sicura.

Hanno il conforto dei giochi e possono anche

passeggiare per strada, guardando i dipinti;

a noi non è concesso neppure vedere la luce: rinchiuse

in casa, siamo consunte da pensieri angosciosi (trad. G. Paduano)].

Il tema, come si vede, è un confronto - dal punto di vista delle donne, e messo in bocca a una donna - fra la vita maschile, che si dispiega liberamente negli spazi aperti e pubblici, e quella femminile mortificata nel buio degli interni domestici: un tema che ha lontane origini euripidee, nella famosa rhesis di Medea (vv. 230 ss.), e che affiora anche altrove in poesia augustea, ${ }^{47}$ per poi conoscere riprese importanti nella letteratura europea, ${ }^{48}$ come nel Proemio del Decamerone di Boccaccio, ${ }^{49}$ nel Don Juan di Byron, ${ }^{50}$ e altrove. Se le 
coincidenze tra Ovidio, Museo e Agazia accreditano l'ipotesi dell'esistenza di un grande modello letterario, perduto, del primo ellenismo (si è pensato perfino a Callimaco) in cui quel tema poteva avere un ruolo centrale,${ }^{51}$ anche l'ode di Orazio può costituire un indizio se non di rinvio a un testo preciso, perlomeno di un interesse diffuso per questo tema.

Ora, per tornare alla domanda posta nel titolo di questa conversazione, si può considerare l'ode 3.12 di Orazio come una sorta di eroide? Se le Heroides - il testo poetico latino che, accanto ai componimenti del Corpus Tibullianum legati al nome di Sulpicia, in maniera più sistematica e consapevole dà spazio alla voce delle donne; o meglio, a quella che il poeta-maschio Ovidio immagina che sia la voce delle donne $-{ }^{52}$ sono anche, fra molte altre cose, una riflessione dolente sulla condizione della donna, sulla sua stessa natura (in confronto con quella maschile) e sulla sua psicologia, allora anche carm. 3.12 può in qualche modo essere assimilata a un'eroide. ${ }^{53}$ Per quanto diversa possa essere la forma e la tecnica letteraria (è chiaro che l'epistolarità in quanto tale accentua nella raccolta di Ovidio il senso di lontananza, di separatezza), non così diversa è l'ideologia che entrambi i testi manifestano: la constatazione amara della minorità sociale e psicologica della donna rispetto all'universo maschile che si legge nell'opera ovidiana, come espressione diretta delle figure femminili protagoniste delle singole epistole, attraverso la loro voce, è la stessa del lamento di Neobule.

Anche Orazio cioè (cui, diversamente da Ovidio, non si accredita un interesse per la psicologia femminile - quello che per altri è un'accusa di curiosità un po' morbosa) sa guardare all'amore dal di fuori, anche con occhi non suoi, e anche con occhi femminili. ${ }^{54}$ La stessa struttura 'senza conclusione' dell'ode sembra voler lasciare una situazione aperta, fluida, e potrebbe in questo senso collegarsi alla scelta di Orazio - che colpisce per il contrasto col modello di Alceo - di lasciare indistinta la voce, di non dirci se a parlare sia Neobule o un'identità maschile, come a sperimentare appunto una mimesi, un'immedesimazione con un'altra identità, con un altro genere. Per questa capacità mimetica, di guardare con occhi femminili, l'ode richiama appunto l'esperienza delle Heroides e di altri testi elegiaci quali quelli legati al ciclo di Sulpicia, non solo le sei elegie attribuite a Sulpicia ma anche i cinque elegidia dell'amico di Sulpicia' che le precedono ([Tib.] 3.8-12). ${ }^{55}$ In questi testi si alterna uno sguardo esterno su Sulpicia e Cerinto $(8,10$ e 12) e uno interno, con la voce stessa di Sulpicia (9 e 11), ma che sembra simulata da qualcun altro al suo posto (quasi la drammatizzazione della concezione della donna quale proiezione delle fantasie e del desiderio maschile), come a sperimentare lo spostamento del punto di vista in tutte le possibili prospettive. ${ }^{56}$ Quasi una spia dell'intenzione di osservare dal di fuori i meccanismi psicologici di una relazione erotica e di sperimentare le varie modalità del discorso amoroso.

Ma è notevole anche che sia istituito un confronto fra il modo di vivere l'eros da parte della donna e da parte dell'uomo ([Tib.] 3.11.17-8 Optat idem iuvenis quod nos, sed tectius optat: / nam pudet haec illum dicere verba palam), ${ }^{57}$ come a conferma dell'intenzione di osservare dall'esterno i meccanismi psicologici di una relazione erotica e le varie modalità del discorso amoroso in funzione del genere, maschile o femminile, dei personaggi che vi sono coinvolti. Naturalmente, in linea con questa intenzione, l'impiego di più voci parlanti drammatizza l'idea della frammentazione dell'esperienza erotica, quasi offrendo al suo lettore l'immagine di un testo diviso fra un codice-norma (quello della società romana) che lo inquadra e un desiderio che ne fuoriesce, che gli si sottrae (quello percepibile nella voce 'elegiaca' di Sulpicia e di chi le si affianca). 
37 alcaica in chiave ellenistica, e con una moderna sensibilità romana - mi pare che possa trovare la sua collocazione più appropriata, e che contribuisca in maniera significativa ad arricchire il profilo di Orazio.

\section{BIBLIOGRAFIA}

Bettini 1986

M. Bettini, Antropologia e cultura romana, Roma 1986

Cairns 1977

F. Cairns, Horace on Other People's Love Affairs (Odes 1.27; 2.4; 1.8; 3.12), in "QU" 24, 1977, 138-47 (= Roman Lyric. Collected Papers on Catullus and Horace, Berlin/Boston 2012, 262-83)

Cavarzere 1996

A. Cavarzere, Sul limitare. Il "motto" e la poesia di Orazio, Bologna 1996

Cavarzere - Aloni - Barchiesi 2001

A. Cavarzere - A. Aloni - A. Barchiesi (edd.), Iambic Ideas. Essays on a Poetic Tradition from Archaic Greece to the Late Roman Empire, Lanham 2001

Citroni 1995

M. Citroni, Poesia e lettori in Roma antica, Roma-Bari 1995

Commager 1962

S. Commager, The Odes of Horace: A Critical Study, New Haven 1962

Esposito 2005

E. Esposito (ed.), Il Fragmentum Grenfellianum (P. Dryton 50), Bologna 2005

Fitzgerald 1995

W. Fitzgerald, Catullan Provocations: Lyric Poetry and the Drama of Position, Berkeley 1995

Fraenkel 1957

E. Fraenkel, Horace, Oxford 1957

Fredericks 1976

S.C. Fredericks, A Poetic Experiment in the Garland of Sulpicia (Corpus Tibullianum, 3, 10), in "Latomus" 35, 1976, 761-82

Hallett 2006

J. Hallett, Sulpicia and her Fama: An Intertextual Approach to Recovering her Latin Literary Image, in “Class. World" 100, 2006, 37-42

Heinze 1960 
R. Heinze, Die horazische Ode, in R. H., Vom Geist des Römertums, Darmstadt 1960, 172-89 (ma già 1923)

Hunter 1996

R. Hunter, Theocritus and the Archaeology of Greek Poetry, Cambridge 1996

Jaeger 1995

M. Jaeger, Reconstructing Rome: the Campus Martius and Horace, Ode 1.8, in “Arethusa” 28, 1995, 177-91

Keith 1997

A. Keith, 'Tandem Venit Amor': A Roman Woman Speaks of Love, in J.P. Hallett - M.B. Skinner (edd.), Roman Sexualities, Princeton 1997, 295-310

Kießling-Heinze 1930

A. Kießling u. R. Heinze (edd.)., Horaz. Oden und Epoden, Berlin 1930

Kißel 1980

W. Kißel, Horaz c. 3, 12 - Form und Gedanke, in “Wien. Stud.” N.F. 14, 1980, 125-32

Korzeniewski 1998

D. Korzeniewski, Metrica greca, trad. it. Palermo 1998

Labate 1993

M. Labate, La forma dell'amore: appunti sulla poesia erotica oraziana, in Atti dei convegni di Venosa, Napoli, Roma, Venosa 1993, 69-87

Laird 1999

A. Laird, Powers of Expression, Expressions of Power. The Speech Presentation in Latin Literature, New York 1999

La Penna 1968

A. La Penna, Orazio e la morale mondana europea, in Orazio, Tutte le opere, Firenze 1968 [= A.L.P., Saggi e studi su Orazio, Firenze 1993, 1-232]

Lardinois - Mcclure 2001

A. Lardinois - L. McClure (edd.), Making Silence Speak: Women's Voices in Greek Literature and Society, Princeton 2001

Marmodoro - Hill 2013

A. Marmodoro - J. Hill (edd.), The author's Voice in Classical and Late Antiquity, Oxford 2013

Milnor 2002

K.L. Milnor, Sulpicia's (Corpo)reality: Elegy, Authorship, and the Body in [Tib.] 3.13, in "ClAnt" 21, 2002, 259-82

Morisi 1999

L. Morisi (ed.), Catullo. Attis (carme LXIII), Bologna 1999

Nicosia 1976

S. Nicosia, Un carme imitato da Orazio, in Tradizione testuale diretta e indiretta dei poeti di Lesbo, Roma 1976, 183-99 
Nielsen 1980

R.M. Nielsen, Horace, Odes III.12: of Longing and Wool Baskets, in C. Deroux (ed.), Studies in Latin Literature and Roman History, vol. 2, Bruxelles 1980, 237-44

Nisbet-Rudd 2004

R.G.M. Nisbet \& N. Rudd (edd.), A Commentary on Horace, Odes, Book III, Oxford 2004

Oliensis 2002

E. Oliensis, Feminine endings, lyric seductions, in D. Feeney \& T. Woodman (edd.), Traditions and Contexts in the Poetry of Horace, Cambridge 2002, 93-106

Pardini 1993

A. Pardini, EME DEILAN (Alc. Fr. 10 V.): ricostruzione, commento, interpretazione complessiva, in "RCCM" 35, 1993, 25-47

Pasquali 1920

G. Pasquali, Orazio lirico, Firenze 1920 (=1966, a c. di A. La Penna)

Pöschl 1991

V. Pöschl, Horazische Lyrik. Interpretationen, Heidelberg 19912, 324-32 (già in V.P., Kleine Schriften I, Heidelberg 1979, 245-53)

Quinn 1980

K. Quinn (ed.), Horace. The Odes, London 1980

Rosati 1996

G. Rosati (ed.), P. Ovidii Nasonis Heroidum epistulae XVIII - XIX (Leander Heroni - Hero Leandro), Firenze 1996

Syndikus 1973

H.P. Syndikus, Die Lyrik des Horaz. Eine Interpretation der Oden, Bd. II, Darmstadt 1973

Timpanaro 1994

S. Timpanaro, Il ius osculi $e$ un passo di Frontone, in S. T., Nuovi contributi di filologia e storia della lingua latina, Bologna 1994, 365-79 (già in "Maia" 39, 1987, 201-11)

Tueller 2008

M.A. Tueller, Look Who's Talking: Innovations in Voice and Identity in Hellenistic Epigram, Leuven 2008 Wilamowitz 1971

U. v. Wilamowitz-Moellendorff, Des Mädchen Klage. Eine alexandrinische Arie, in Kleine Schriften, vol. 2, Berlin-Amsterdam 1971, 95-120 (già in "Nachr. K. Gesell. Wiss. Göttingen, phil.-hist. Kl., 1896, 209-32)

Wyke 2002

M. Wyke, The Roman Mistress. Ancient and Modern Representations, Oxford 2002

Zeitlin 1996

F. Zeitlin, Playing the Other. Gender and Society in Classical Greek Literature, Chicago and London 1996 


\section{NOTE}

1. Oltre ai principali commenti (soprattutto Kießling-Heinze 1930, Syndikus 1973, Quinn 1980, Nisbet-Rudd 2004), cfr. Nicosia 1976, Cairns 1977, Kißel 1980, Nielsen 1980, Pöschl 1991.

2. Così Kießling-Heinze 1930, 314; cfr. anche Kißel 1980, 127.

3. Cfr. Korzeniewski 1998, 115 n. 57. È interessante che alla stessa matrice degli ionici veniva ricondotto, secondo una teoria attestata da Efestione (ampia illustrazione della discussa questione in Morisi 1999, 49 ss.), anche il galliambo, il metro del catulliano carme 63, di Attis.

4. Cfr. Wilamowitz 1971, 112 n. 2.

5. Pasquali 1920, 86-7 e 103; cfr. anche Fraenkel 1957, 178.

6. Ben illustrata da Cavarzere 1996, 229 n. 36, che discute le diverse proposte.

7. Pöschl 1991, 325.

8. Cairns 1977,138 s.

9. Citroni 1995, 350 n. 6.

10. Lo pensava ad es. Heinze 1960, 176.

11. Sul testo di Alceo rinvio alla dettagliata analisi di Pardini 1993, il quale avanza l'ipotesi che si tratti di una sorta di parodia, di un lamento d'amore ironico, messo in bocca alla promessa sposa di Pittaco (46).

12. Così La Penna 1968, CXVIII $(=1993,149)$.

13. Cfr. Syndikus 1973, 131-4.

14. Di "odd and almost fragmentary Ode" parla Commager 1962, 143.

15. Cfr. Pasquali 1920, 87 ss.

16. Cfr. Cairns 1977, 140-4.

17. Il grecismo è accentuato dalla desinenza greca, la -e lunga finale che è un ablativo della forma della I declin. Bellerophontes.

18. Cairns 1977, 144-5.

19. Sull'espressione patruae verbera linguae Timpanaro 1994, 376 n. 24, sollevò infatti, anni fa, un problema esegetico: mentre generalmente si intende con l'espressione un riferimento alle metaforiche 'sferzate' morali, alle aspre critiche dello zio tutore della moralità della nipote (cfr. ad es. Bettini 1986, 33), egli voleva vedervi anche un riferimento concreto all'osculum, il bacio 'inferto' dallo zio alla nipote non solo come testimonianza di affetto parentale (quale attestazione di parentela cui la donna era soggetta da parte dei parenti maschi fino al sesto grado: è questo propriamente il ius osculi), ma anche come mezzo per controllare l'osservanza del divieto del vino (vino che nell'ode è menzionato appena prima). Lingua sarebbe quindi “non l'organo del parlare, ma dell'ispezionare la bocca di Neobùle" (Timpanaro), e verbera quindi i colpi concreti, materiali (e un po' ripugnanti) nella loro fisicità, di questa 'ispezione'. Indipendentemente dalla dubbia plausibilità di questa interpretazione è evidente che verbera linguae implica comunque la constatazione di una violenza sociale inferta alla donna dalla lingua maschile, l'organo della comunicazione e il simbolo delle convenzioni e dei pregiudizi di una società maschilista che controlla la moralità della donna e inibisce la sua libertà in amore.

20. Da leggere Bettini 1986, 27-49.

21. Ad es. 8.1 e 10, 76.12 e 19 etc.

22. Cfr. Nisbet-Rudd 2004, ad loc.

23. Cfr. Quinn 1980, 266.

24. Cfr. Timpanaro 1991, 377 n. 24, che corregge Pasquali 1920, 87. Solo nella cultura cristiana si diffonderà la metafora, che era stata già di Terenzio, del 'lavar via' il peccato, la colpa; mentre il 'lavare col vino' è limitato a rare occorrenze tecniche o a contesti sacrali (Verg. Aen. 6.227). Qui, com'è ovvio, il carattere letterario emerge anche nell'implicita associazione dell'immagine del 'lavare' con l'idea del vino come elemento liquido, e nel presupporre l'idea di Bacco Lyaeus, del vino che 'scioglie' gli affanni (i verbi greci $\lambda u ́ \omega$ 'sciolgo' e $\lambda$ oúw 'lavo', come i latini luo e lavo, 
hanno la stessa origine), un'idea sfruttata in poesia per concettismi come Prop. 3.5.21 multo mentem vincire Lyaeo (cioè 'legare' la mente con Bacco 'che scioglie'), o lo stesso Hor. carm. 1.7.22 s. uda Lyaeo / tempora populea fertur vinxisse corona.

25. Oltre all'epodo 11 dello stesso Orazio, cfr. ad es. [Verg.] Ciris 177 ss. (la protagonista innamorata) nulla colum novit, carum non respicit aurum, / non arguta sonant tenui psalteria chorda, / non Lybico molles plauduntur pectine telae...; o Verg. ecl. 2.70 s.; Sen. Phaedr. 103, etc.

26. Cfr. già Kießling-Heinze ad loc., e poi Nisbet-Rudd 2004, 165.

27. Cfr. Esposito 2005.

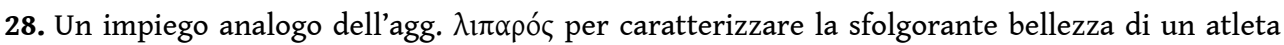
percepita da occhi femminili è nel già richiamato secondo idillio teocriteo: cfr. i vv. 51 e 103 ma anche 133.

29. Così Cairns 1977, 144.

30. Pasquali 1920, 95: “il dio [...] è un'espressione simbolica della bellezza giovenile di Ebro".

31. L'antitesi fra attività venatoria e eros ha notoriamente il suo archetipo nell'Ippolito euripideo.

32. Penso ad es. all'ode 1.8 dello stesso Orazio, dove il poeta si rivolge a Lidia che ha sedotto il giovane Sibari, il quale non si dedica più alle attività atletiche e agli esercizi militari.

33. Di “Seelenbewegung des Mädchen" parla Pöschl 1991, 326.

34. Cfr. Oliensis 2002.

35. Funzione simile ha il nome di Enipeo, un altro fiume, per il giovane nuotatore e cavaliere ammirato dalla Asterie dell'ode 3.7; e probabilmente anche quello di Sibari in 1.8 (Jaeger 1995, $184 \mathrm{~s})$. Le rive del Tevere erano di fatto un luogo quasi topico per le esibizioni di abilità natatoria dei giovani romani (Cic. Cael. 36 habet hortos ad Tiberim ac diligenter eo loco paratos quo omnis iuventus natandi causa venit), che dovevano sollecitare una specie di voyeurismo femminile (il tema sembra affiorare anche da Ov. her. 18.95-6 Nunc etiam nando dominae placuisse laboro, / atque oculis iacto bracchia nostra tuis: cfr. Rosati 1996, ad loc.).

36. "Das Gedicht hat denn auch keinen eigentlichen Schluß - sowenig wie der Kreislauf von Neobules Sinnen": Kießling-Heinze 1930, 314.

37. Secondo alcuni implicherebbe un'associazione all'idea di 'nuovi progetti', cioè un tentativo di uscire dal mondo chiuso in cui è stata finora confinata per avventurarsi verso una vita nuova e diversa, 'aperta'.

38. Sull'idea del giambo come frusta-invettiva, molto in Cavarzere-Aloni-Barchiesi 2001 (ad es. 219-21).

39. Il nome stesso della donna deve avere una funzione antonomastica come quella che ha il nome del padre (cfr. ad es. Hor. epod. 6.13; epist. 1.19.25; Ov. Ib. 54; Mart. 7.12.6).

40. "Io non sono come tu mi hai resa famosa" suona la protesta delle Licambidi contro Archiloco che si legge in un epigramma di Dioscoride (AP 7.351): mentre in un altro epigramma di dubbia attribuzione (AP 7.352 = Suppl. Hell. 997) esse si dicono vittime di "una guerra contro le donne" (6) da parte di "un uomo senza onore" (8).

41. L'attenzione alla 'voce' nei testi antichi si è imposta come un tema importante negli studi degli ultimi decenni: qui mi limito a segnalare pochi lavori recenti dove si potrà trovare ampia bibliografia: Zeitlin 1996; Laird 1999; Lardinois - Mcclure 2001; Tueller 2008; Marmodoro - Hill 2013.

42. Wyke 2002, 156 .

43. Cfr. Fitzgerald 1995.

44. Si veda ad es. la discussione in Wyke 2002, 155-91.

45. Cfr. Zeitlin 1996, 363 "the final paradox may be that theater uses the feminine for the purposes of imagining a fuller model for the masculine self, and 'playing the other' opens that self to those often banned emotions of fear and pity"; ma tutto il lavoro è importante su questo aspetto.

46. Sui rapporti tra questi due testi cfr. spec. Hunter 1996, 8-10. 
47. Si veda ad es. l'opposizione fra natura femminile e natura maschile, con un elenco delle attività atletiche e sportive consuete nella vita dei giovani romani del tempo, che ancora in Ovidio si legge in ars 3.381 ss. hos ignava iocos tribuit natura puellis; / materia ludunt uberiore viri. / Sunt illis celeresque pilae iaculumque trochique / armaque et in gyros ire coactus equus. / Nec vos Campus habet nec vos gelidissima Virgo / nec Tuscus placida devehit amnis aqua.

48. Cfr. Rosati 1996, 20 n. 36.

49. Proem. E oltre a ciò, ristrette da' voleri, da' piaceri, da' comandamenti de' padri, delle madri, de' fratelli e de' mariti, il più del tempo nel piccolo circuito delle loro camere racchiuse dimorano, e quasi oziose sedendosi, volendo e non volendo in una medesima ora, seco rivolgono diversi pensieri, li quali non è possibile che sempre sieno allegri. E se per quegli alcuna malinconia, mossa da focoso disio, sopravviene nelle lor menti, in quelle conviene che con grave noia si dimori, se da nuovi ragionamenti non è rimossa: senza che elle sono molto men forti che gli uomini a sostenere; il che degli innamorati uomini non avviene, sì come noi possiamo apertamente vedere. Essi, se alcuna malinconia o gravezza di pensieri gli affligge, hanno molti modi da alleggiare o da passar quello; per ciò che a loro, volendo essi, non manca l'andare attorno, udire e veder molte cose, uccellare, cacciare, pescare, cavalcare, giucare o mercatare; de' quali modi ciascuno ha forza di trarre, o in tutto o in parte, l'animo a sé e dal noioso pensiero rimuoverlo almeno per alcuno spazio di tempo, appresso il quale, o in un modo o in uno altro, o consolazion sopravviene o diventa la noia minore.

50. I 194 Man's love is of man's life a thing apart, / 'tis woman's whole existence; man may range / the court, camp, church, the vessel, and the mart; / sword, gown, gain, glory, offer in exchange / pride, fame, ambition, to fill up his heart, / and few there are whom these cannot estrange; / men have all these resources, we but one / to love again, and be again undone.

51. Sulla questione rinvio a Rosati 1996, 12-15.

52. Una discussione approfondita su meccanismi e implicazioni del "taking the woman's part" nell'elegia latina si legge in Wyke 2002, 155-91.

53. Di diverso avviso Nisbet-Rudd 2004, 165: "Instead of showing the empathy with women that we find in Virgil and sometimes in Ovid, Horaces concentrates on the vigour of the male athlete"; 166: "Hebrus, the true subject of the poem".

54. Cfr. su questo punto Labate 1993, 87, il quale osserva che Orazio sente come "pertinente alla voce lirica la capacità di interpretare anche soggettività diverse da quella del poeta: punti di vista cui l'io lirico può avvicinarsi simpateticamente, o con cui gli è concesso talvolta di immedesimarsi mimeticamente" (con specifico rifermento a 3.12, e dopo l'illustrazione di quelle odi, 1.25 e 1.23, in cui Orazio si colloca nel punto di vista della donna).

55. Non tutti gli studiosi peraltro condividono la tesi dei due autori distinti: cfr. Hallett 2006.

56. In particolare 3.10 sembra voler impedire l'identificazione del parlante, lasciare il lettore nell'ambiguità, e come costringerlo a passare da un'ipotesi all'altra: cfr. Fredericks 1976, e Milnor 2002, 268-9.

57. Su questo tema è importante Keith 1997.

\section{RIASSUNTI}

L'ode 3.12 di Orazio, tra le meno studiate del poeta, presenta alcune singolarità formali e tematiche (la 'simulazione' della voce femminile, il lamento per la condizione di vita della donna, privata dei piaceri concessi ai coetanei maschi, etc.) che per certi aspetti la assimilano alle 
Heroides di Ovidio. L'ode, un originale sviluppo di un testo-modello di Alceo a noi pervenuto solo in forma frammentaria, e la cui protagonista, Neobule, richiama un famoso personaggio di Archiloco, mette in scena un confronto culturale tra mondo greco e mondo romano, sul quale si innesta un altro, marcato confronto di gender, di genere sessuale. Il tema presenta vistosi e importanti paralleli con altri autori latini e greci (da Ovidio a Museo fino ad Agazia, per poi passare ad alcuni grandi testi della letteratura europea) e sembra rinviare a una sua diffusa presenza, solo parzialmente documentata, nella cultura letteraria ellenistica. La tematica amorosa, e l'adozione di un punto di vista (se non della stessa voce) femminile, accostano quest'ode oraziana all'elegia erotica latina, e in particolare a Sulpicia, aprendo uno spiraglio sull'universo del 'desiderio femminile' che arricchisce sensibilmente la nostra immagine di Orazio.

\section{INDICE}

Mots-clés : Alceo, Archiloco, Ovidio, Heroides, Sulpicia, voce, elegia latina, gender, vino, patruus, caccia, socialità, piacere, seduzione, desiderio femminile

\section{AUTORE}

\section{GIANPIERO ROSATI}

Scuola Normale Superiore 